\title{
Enhanced Near-Infrared Electroluminescence from a Neodymium Complex in Organic Light-Emitting Diodes with a Solution-Processed Exciplex Host
}

\author{
Afshin Shahalizad, ${ }^{1}$ Dae-Hyeon Kim, ${ }^{2}$ Sanyasi Rao Bobbara, ${ }^{1}$ Youichi Tsuchiya,, 3 \\ Anthony D'Aléo, ${ }^{4,5}$ Chantal Andraud, ${ }^{6}$ Jean-Charles Ribierre, ${ }^{2,3, a)}$ Jean-Michel \\ Nunzi, ${ }^{1, b)}$ and Chihaya Adachi ${ }^{2,3, c)}$ \\ ${ }^{1}$ Department of Physics, Engineering Physics and Astronomy, Queen's University, Kingston, Ontario K7L 3N6, Canada \\ ${ }^{2}$ Center for Organic Photonics and Electronics Research (OPERA), Kyushu University, Fukuoka, Japan \\ ${ }^{3}$ Japan Science and Technology Agency (JST), ERATO, Adachi Molecular Exciton Engineering Project, Fukuoka, Japan \\ ${ }^{4}$ Aix Marseille Univ, CNRS, CINaM UMR 7325, Campus de Luminy, Case 913, Marseille, France \\ ${ }^{5}$ Building Blocks for Future Electronics Laboratory (2-B FUEL), The joint CNRS-Ewha-Yonsei Laboratory, UMI 2002, \\ Seoul, Republic of Korea \\ ${ }^{6}$ Univ Lyon, Ens de Lyon, CNRS UMR 5182, Université Claude Bernard Lyon 1, Laboratoire de Chimie, F69342, Lyon, \\ France
}

We report the enhanced near-infrared (NIR) electroluminescence from a $\mathrm{Nd}^{3+}$-complex with thenoyltrifluoroacetone and 1,10phenanthroline ligands. The NIR-emitting complex was blended into an exciplex-forming co-host system comprising 2,7bis(diphenylphosphoryl)-9,9'-spirobifluorene as the electron transport material and 4,4',4"-tris(carbazol-9-yl)triphenylamine as the hole transport material, in solution-processed small molecule organic light-emitting diodes (OLEDs). This binary ambipolar host system favors direct charge trapping and exciton formation on the $\mathrm{Nd}^{3+}$-complex molecules. Efficient energy transfer from the singlet and triplet exciplexes formed between the host molecules to the $\mathrm{Nd}^{3+}$ ions contributes to the enhanced luminescence efficiency. The photoluminescence quantum yield of this blend is $1.2 \%$, and the optimized OLED shows a maximum electroluminescence external quantum efficiency of $0.034 \%$. The device also exhibits a low efficiency roll-off of only $12 \%$ over a current density range of $100 \mathrm{~mA} / \mathrm{cm}^{2}$, due to the reduced triplet-polaron annihilation.

Light-emitting lanthanides have been increasingly researched in recent years, motivated by their use in technologies such as biomedical imaging and plastic optoelectronics. ${ }^{1,2} \mathrm{Nd}^{3+}, \mathrm{Yb}^{3+}$, and $\mathrm{Er}^{3+}$ lanthanide ions possess long-lived and narrow emission bands in their near-infrared (NIR) spectra (900-1600 nm). Because of this, these lanthanide ions have been widely studied in modern telecommunication systems and organic solid-state amplifiers and lasers, which mostly employ external optical sources for exciting lanthanides. ${ }^{3-5}$

The development of electrically-pumped organic light sources based on NIR lanthanides is particularly attractive. This is because very compact light sources driven by electrical excitation can be fabricated. Such light sources can be integrated into various lab-on-chip platforms. ${ }^{6}$ Despite their potential in many applications, reports on lanthanide-based NIR organic light-emitting diodes (OLEDs) with electroluminescence (EL) in the telecommunication wavelength range $(900-1600 \mathrm{~nm})$ are scarce. ${ }^{7-10}$ Furthermore, thermal evaporation has been extensively used to fabricate NIR OLEDs. However, lanthanide complexes have been shown to readily decompose at the temperatures required for deposition, resulting in poor device efficiency. ${ }^{11}$ For this reason, solution-processing is an attractive technique for

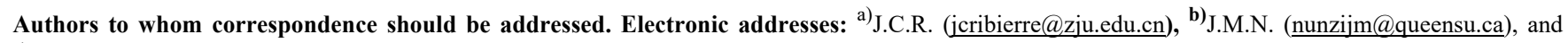
${ }^{c}$ C.A. (adachi@cstf.kyushu-u.ac.jp).
} 
incorporating lanthanide complexes into organic host materials, which would eventually enable the realization of efficient, inexpensive, and easy-to-manufacture NIR OLEDs.

We previously reported the NIR emission of $\mathrm{Nd}(\mathrm{TTA})_{3}$ Phen complex containing thenoyltrifluoroacetone (TTA) and 1,10-phenanthroline (Phen) ligands, which was doped into 1,3-bis(9-carbazolyl)benzene $(\mathrm{mCP})$ in solution-processed small molecule NIR OLEDs. ${ }^{12}$ The device containing $20 \mathrm{wt} . \%$ of the complex emitted light only at the three characteristic luminescence peaks of $\mathrm{Nd}^{3+}$, and exhibited an external quantum efficiency (EQE) as high as $0.022 \%$. This EQE was the highest reported value for solution-processed NIR OLEDs based on $\mathrm{Nd}^{3+}$-complexes but it should still be improved for practical applications.

In this letter, we demonstrate an EL efficiency enhancement from $\mathrm{Nd}(\mathrm{TTA})_{3}$ Phen doped into a solutionprocessable exciplex-forming small molecule binary host system. The host system consists of the 2,7bis(diphenylphosphoryl)-9,9'-spirobifluorene (SPPO13) electron transporting material and the 4,4',4"tris(carbazol-9-yl)triphenylamine (TCTA) hole transporting material. The enhanced EL is due to the wellbalanced ambipolar charge transport properties of the emissive layer (EML), and precise control of the excitonic processes occurring during device operation. Furthermore, we show that the fabricated NIR OLED exhibits a very low EQE roll-off, which is an important requirement for practical applications of electrically-driven organic devices based on lanthanide materials. ${ }^{2,6}$

The device structure of the fabricated NIR OLED is as follows: ITO/PEDOT:PSS (35 nm)/EML (20 $\mathrm{nm}) / \mathrm{TPBi}(40 \mathrm{~nm}) / \mathrm{LiF}(0.8 \mathrm{~nm}) / \mathrm{Al}(100 \mathrm{~nm})$, where the composition of the EML is SPPO13:TCTA: $\operatorname{Nd}(\mathrm{TTA})_{3}$ Phen $\quad(6: 3: 1 \quad$ weight ratio). In this device, poly(3,4ethylenedioxythiophene):poly(styrenesulfonate) (PEDOT:PSS) and 2,2',2"-(1,3,5-benzinetriyl)-tris(1phenyl-1-H-benzimidazole) (TPBi) are used as the hole injection layer and electron injection layer, respectively. The chemical structures of the materials used in the device are shown in Fig. S1 in the supplementary material. Except for the EML, details of the fabrication process and device characterizations are similar to those in our previous work. ${ }^{12}$ SPPO13 and TCTA are employed for their high charge carrier mobility and solubility in common organic solvents that enables fabrication of highquality thin films. ${ }^{13-15}$ The EML was deposited on top of the PEDOT:PSS layer, according to the procedure by Chen et $_{\text {al. }}{ }^{13}$ 
Atomic force microscope (AFM) images of SPPO13:TCTA and SPPO13:TCTA:Nd(TTA) ${ }_{3}$ Phen films prepared by spin-coating on quartz substrates are shown in Fig. S2 in the supplementary material. These images show very smooth surface morphologies for the doped and undoped solution-processed films, with low root-mean-square (RMS) roughness vales $(\leq 0.4 \mathrm{~nm})$. This suggests that no recrystallization or hostguest phase segregation occurs during thermal annealing.

The EL spectrum of the device is shown in Fig. 1. It is worth noting that we did not observe any EL by our naked eyes, implying that the NIR OLED does not exhibit any emission in the visible region. The EL spectrum displayed in Fig. 1 contains only the three characteristic NIR emission peaks from $\mathrm{Nd}^{3+}$ at 890 $\mathrm{nm}\left({ }^{4} F_{3 / 2} \rightarrow{ }^{4} I_{9 / 2}\right), 1060 \mathrm{~nm}\left({ }^{4} F_{3 / 2} \rightarrow{ }^{4} I_{11 / 2}\right)$, and $1330 \mathrm{~nm}\left({ }^{4} F_{3 / 2} \rightarrow{ }^{4} I_{13 / 2}\right)$ without any emission in the visible spectral region. It is well known that $f-f$ transitions in lanthanides are Laporte-forbidden, so they possess very low molar absorption coefficients, which makes the direct excitation of lanthanides inefficient. ${ }^{16}$ Thus, lanthanides are coordinated to organic ligands that can be excited by optical or electrical pumping. The ligands then transfer their excitation energies to the lanthanide ions via energy transfer mechanisms in a process called antenna effect. ${ }^{17}$

Steady-state photoluminescence (PL) spectra of SPPO13, TCTA, SPPO13:TCTA, and SPPO13:TCTA: $\operatorname{Nd}(\text { TTA })_{3}$ Phen blend systems are shown in Fig. 2. The PL spectra of SPPO13 and TCTA significantly overlap with the absorption spectrum of the TTA ligand in $\mathrm{Nd}(\mathrm{TTA}){ }_{3}$ Phen. This suggests that the singlet excited state energies of the host materials can be efficiently transferred to the TTA ligand via Förstertype energy transfer process. The efficient Dexter-type energy transfer from the triplet excited-states of the host materials to those of the Phen and TTA ligands is also expected to occur. However, the PL of SPPO13:TCTA blend is completely different from those of SPPO13 and TCTA. The SPPO13:TCTA blend, which has been previously found to be an exciplex-forming electron donor-acceptor system, ${ }^{18,19}$ exhibits a broad red-shifted emission peak centered at $479 \mathrm{~nm}$. The PL spectrum of the SPPO13:TCTA:Nd(TTA) ${ }_{3}$ Phen blend shows a maximum at $467 \mathrm{~nm}$. We attribute this small 12-nm blueshift to the perturbation of the exciplex interaction between TCTA and SPPO13 by Nd(TTA) ${ }_{3}$ Phen. The energy gap between the lowest unoccupied molecular orbital (LUMO) of SPPO13 and the highest occupied molecular orbital (HOMO) of $\mathrm{Nd}(\mathrm{TTA}){ }_{3}$ Phen is smaller than the HOMO-LUMO gap of SPPO13 or Nd(TTA) 3 Phen (see Fig. 4), as is required for exciplex formation. ${ }^{20,21}$ Despite this, we did not observe any exciplex emission from the SPPO13: $\mathrm{Nd}(\mathrm{TTA})_{3}$ Phen blend. This may be because the molecular structure of the complex is spherical, thus hindering exciplex formation in the SPPO13:Nd(TTA) ${ }_{3}$ Phen system. Consequently, because no signature of the PL spectra of individual 
SPPO13 and TCTA is seen in the PL spectrum of the SPPO13:TCTA blend, they cannot contribute to the excitation process of $\mathrm{Nd}(\mathrm{TTA})_{3}$ Phen in the SPPO13:TCTA:Nd(TTA) ${ }_{3}$ Phen blend system. For this reason, we expect that only SPPO13:TCTA singlet and triplet exciplexes contribute to the sensitization of the $\mathrm{Nd}^{3+}$ ion in the complex. In such a case, the singlet and triplet exciplex energy levels are very close to each other. ${ }^{22}$ Fig. S3 in the supplementary material shows that their energy can be transferred to the triplet energy levels of the ligands and subsequently to the $\mathrm{Nd}^{3+}$ ions. Direct energy transfer from the singlet and triplet exciplexes to the $\mathrm{Nd}^{3+}$ ions can also occur. The SPPO13:TCTA exciplexes are also expected to form under electrical excitation in the device, contributing to the sensitization of the $\mathrm{Nd}^{3+}$ ions. In contrast to the PL spectra, we did not detect any visible EL emission from the host materials and the SPPO13:TCTA exciplexes. This indicates that energy transfer from exciplexes to the NIR-emitting complex is efficient, and that direct formation of excitons on $\operatorname{Nd}(\mathrm{TTA})_{3}$ Phen molecules occurs efficiently in the device. The latter process can be understood when considering the energy level alignments between the electron transport and hole transport layers (ETL and HTL) and the EML in the device structure, as shown in Fig. 3.

To elucidate the formation of exciplexes and to better understand the sensitization mechanism of the $\mathrm{Nd}^{3+}$ ion in the complex, we performed steady-state and transient PL measurements in the visible spectral region for SPPO13, TCTA, SPPO13:TCTA, and SPPO13:TCTA:Nd(TTA) 3 Phen. The transient measurements were carried out using the time-correlated single-photon counting (TCSPC) technique, and time-resolved fluorescence plots are shown in Fig. S4 in the supplementary material. In the SPPO13:TCTA system, short and long exciplex fluorescence decays are observed with lifetimes of $53 \mathrm{~ns}$ and $297 \mathrm{~ns}$, respectively. These emission lifetimes are much longer than those of SPPO13 and TCTA (see supplementary material for a detailed explanation). In the SPPO13:TCTA:Nd(TTA) ${ }_{3}$ Phen system, the short and long decay exciplex fluorescence lifetimes are $7.7 \mathrm{~ns}$ and $88.4 \mathrm{~ns}$, respectively, which are noticeably shorter than those in the SPPO13:TCTA system. This is indeed consistent with the SPPO13:TCTA exciplex energies being effectively transferred to the $\mathrm{Nd}^{3+}$ ions in the SPPO13:TCTA:Nd(TTA) ${ }_{3}$ Phen blend. A NIR photoluminescence quantum yield (PLQY) as high as $1.2 \%$ is measured in the SPPO13:TCTA:Nd(TTA) $)_{3}$ Phen system. Note that this PLQY is higher than the reported values $(0.4 \%$ and $0.5 \%$ ) using the same complex dispersed in the mCP host. ${ }^{12}$ The higher PLQY obtained in the present study is attributed to the more efficient sensitization of the $\mathrm{Nd}^{3+}$ ions by the singlet and triplet exciplexes (see also FIG. S5 in the supplementary material). 
Current density vs. voltage and external quantum efficiency (EQE) vs. current density plots of the device are shown in Fig. 4. The device starts emitting NIR light at about $11 \mathrm{~V}$. This turn-on voltage is very close to the reported values in our previous work, ${ }^{12}$ and is lower than reported values for $\mathrm{Nd}^{3+}$ complex-based OLEDs, which range from $12 \mathrm{~V}$ to $30 \mathrm{~V} \cdot{ }^{23-28} \mathrm{~A}$ maximum EQE as high as $0.034 \%$ is obtained at 130 $\mathrm{mA} / \mathrm{cm}^{2}$, which is $50 \%$ higher than the reported EQE $(0.022 \%)$ for solution-processed $\mathrm{Nd}^{3+}$-based OLEDs with a mCP host. ${ }^{12}$ Except for the thermally-processed $\mathrm{Nd}^{3+}$-based OLEDs reported by Chen et al., ${ }^{28}$ EQE in the present study is larger than any reported EQE for solution-processed or thermally-processed NIR OLEDs based on $\mathrm{Nd}^{3+}$-complexes. ${ }^{8-10,23-27}$ This EQE is also comparable or higher than the EQE values recently reported for NIR OLEDs with EL in the telecommunication wavelength range (900-1600 nm), which were fabricated from thermally-activated delayed fluorescence (TADF) materials $(0.037 \%)^{29}$ or carbon nanotube emitters $(0.014 \%) .{ }^{30}$ It should be mentioned that low efficiency and relatively high working voltages in lanthanide-based NIR OLEDs are mainly caused by the low electroactivity of lanthanide complexes and coupling of the NIR emission to highly vibrating $\mathrm{C}-\mathrm{H}$ and $\mathrm{O}-\mathrm{H}$ bonds present in the ligands and organic materials used in the device structures. ${ }^{12,31}$ In the case of our device, considering the rather large difference in the HOMO levels, the observation of the EL onset above $10 \mathrm{~V}$ is presumably due to strong charge carrier trapping by the $\mathrm{Nd}^{3+}$-complex in the SPPO13:TCTA host. Detailed explanation of the EQE measurement is given in the supplementary material.

Importantly, the EQE only decreases from $0.034 \%$ at $130 \mathrm{~mA} / \mathrm{cm}^{2}$ to $0.030 \%$ at $230 \mathrm{~mA} / \mathrm{cm}^{2}$. This indicates that the device exhibits low efficiency roll-off of only 12\% over a current density range of 100 $\mathrm{mA} / \mathrm{cm}^{2}$. It is known that lanthanide-based OLEDs suffer from triplet-triplet (T-T) and triplet-polaron (TP) annihilation processes. ${ }^{12,32-35}$ As can be seen from Fig. S6, the normalized experimental EQE fits the T-P model developed by Adachi et al. (see supplementary material for details on the T-P and T-T models). ${ }^{32,36}$ In an OLED with a single host, electron and hole polarons are accumulated at the HTL/EML and ETL/EML interfaces because of large energy barriers between the organic layers. As a result, accumulation of charge polarons at the interfaces limits the emission (exciton recombination) zone to very narrow regions close to the interfaces, which subsequently causes severe efficiency roll-off due to quenching of radiative excitons by polarons. Such an effect is particularly observed under large current densities. ${ }^{37-39}$ However, since exciplex emission is a barrier-free process occurring at the donor/acceptor interface and exciplex energy can be transferred to the emitter in a host-guest system, the emission zone is extended to the entire EML, reducing T-P annihilation. ${ }^{40,41}$ Further, balanced charge transport provided by the mixed host in an exciplex-forming system extends the emission zone in the EML as well. ${ }^{42-44}$ In this context, we attribute the low efficiency roll-off in the present study to a reduction of the quenching 
by T-P annihilation. This is due to the small energy barriers at the HTL/EML and ETL/EML interfaces, as well as the extension of emission zone to the entire EML by balanced charge transport provided by the SPPO13:TCTA co-host system and barrier-free energy transfer from the exciplexes to the NIR emitter.

In summary, employing an exciplex-forming binary host comprising solution-processable SPPO13 and TCTA, we demonstrate $50 \%$ enhancement in the EQE from $\mathrm{Nd}(\mathrm{TTA})_{3}$ Phen complex in a NIR OLED. This EQE (0.034\%) is the highest reported for solution-processed NIR OLEDs based on lanthanidecomplexes. The higher EQE obtained in the present study compared with our previous studies is due to the efficient energy transfer from the singlet and triplet SPPO13:TCTA exciplexes to the lanthanide complex, and also due to the better charge transport properties of the mixed host of SPPO13 and TCTA. Moreover, the device exhibits very low efficiency roll-off due to reduced T-P annihilation. This is explained by small energy barriers between the organic layers, balanced charge transport provided by the mixed host, and extension of the emission zone into the entire EML due to barrier-free energy transfer from the exciplexes to the NIR emitter.

\section{SUPPLEMENTARY MATERIAL}

See supplementary material for the chemical structures of the materials in the device, AFM images, transient PL characteristics, and details of the EQE measurement.

\section{ACKNOWLEDGMENTS}

Research at Queen's University was supported by the Natural Sciences and Engineering Research Council (NSERC), the Discovery Grants program (No. RGPIN-2015-05485), and the CREATE program (Novel

Chiral Materials: An International Effort in Research and Education). Research at Kyushu University was supported by the Japan Science and Technology Agency (JST), ERATO, Adachi Molecular Exciton Engineering Project, under JST ERATO Grant Number JPMJER1305, Japan. 


\section{REFERENCES}

${ }^{1}$ S. U. Cho, D. P. Riordan, P. Ciepla, K. S. Kocherlakota, J. K. Chen, and P. B. Harbury, Nat. Chem. Bio. 14, 15 (2018).

${ }^{2}$ H. Q. Ye, Z. Li, Y. Peng, C. C. Wang, T. Y. Li, Y. X. Zheng, A. Sapelkin, G. Adamopoulos, I. Hernández, P. B. Wyatt, and W. P. Gillin, Nat. Mater. 13, 382 (2014).

${ }^{3}$ H. Lin, T. Yu, M.-K. Tsang, G. Bai, Q. Zhang, and J. Hao, Appl. Phys. Lett. 108, 041902 (2016).

${ }^{4}$ J. Xu, S. Tanabe, A. D. Sontakke, and J. Ueda, Appl. Phys. Lett., 107, 081903 (2015).

${ }^{5}$ F. Monteiro, T. Guerreiro, B. Sanguinetti, and H. Zbinden, Appl. Phys. Lett. 103, 051109 (2013).

${ }^{6}$ C. Grivas and M. Pollnau, Lasers \& Photonics Rev. 6, 419 (2012).

${ }^{7}$ K. Jinnai, R. Kabe, and C. Adachi, Chem. Commun. 53, 5457 (2017).

${ }^{8}$ H. Wei, G. Yu, Z. Zhao, Z. Liu, Z. Bian, and C. Huang, Dalton. Trans. 42, 8951 (2013).

${ }^{9}$ S. Penna, A. Reale, R. Pizzoferrato, G. M. Tosi Beleffi, D. Musella, and W. P. Gillin, Appl. Phys. Lett. 91, 021106 (2007).

${ }^{10}$ F. X. Zang, W. L. Li, Z. R. Hong, H. Z. Wei, M. T. Li, and X. Y. Sun, Appl. Phys. Lett. 84, 5115 (2004).

${ }^{11}$ T. Sano, M. Fujita, T. Fujii, and Y. Hamada, Jpn. J. Appl. Phys., 34 (1995) 1883.

${ }^{12}$ A. Shahalizad, A. D’Aléo, C. Andraud, M. H. Sazzad, D.-H. Kim, Y. Tsuchiya, J.C. Ribierre, J.M. Nunzi, and C. Adachi, Org. Electron. 44, 50 (2017).

${ }^{13}$ J. Chen, C. Shi, Q. Fu., F. Zhao, Y. Hu, Y. Feng, and D. Ma, J. Mater. Chem., 22, 5164 (2012).

${ }^{14}$ J.-W. Kang, S.-H. Lee, H.-D. Park, W.-IK. Jeong, K.-M. Yoo, Y.-S. Park, and J.-J. Kim, Appl. Phys. Lett., 90, 223508 (2007).

${ }^{15}$ X. Du, X. Yang, J. Zhao, H. Lin, C. Zheng, and S. Tao, Org. Electron. 38, 344 (2016).

${ }^{16}$ A. D’Aléo, A. Picot, A. Beeby, J. A. G. Williams, B. Le Guennic, C. Andraud, and O. Maury, Inorg. Chem., 47, 10258 (2008).

${ }^{17}$ A. D'Aléo, F. Pointillart, L. Ouahab, C. Andraud, and O. Maury, Coord. Chem. Rev., 256, 1604 (2012).

${ }^{18}$ Y-S. Park, S. Lee, K-H. Kim, S-Y. Kim, J-H. Lee, and J-J. Kim, Adv. Funct. Mater. 23, 4914 (2013).

${ }^{19}$ X. Liu, B. Yao, Z. Zhang, X. Zhao, B. Zhang, W-Y. Wong, Y. Cheng, and Z. Xie, J. Mater. Chem. C, 4, 5787 (2016).

${ }^{20}$ D.-Y. Zhou, L.-S. Cui, Y.-J. Zhang, L.-S. Liao, and H. Aziz, Appl. Phys. Lett. 105, 153302 (2014).

${ }^{21}$ W. Song and J. Y. Lee, Appl. Phys. Lett. 106, 123306 (2015).

${ }^{22}$ K. Goushi, K. Yoshida, K. Sato, and C. Adachi, Nat. Photon. 6, 253 (2012).

${ }^{23}$ L. H. Slooff, A. Polman, F. Cacialli, R. H. Friend, G. A. Hebbink, F. C. J. M. van Veggel, and D. N. Reinhoudt, Appl. Phys. Lett., 78, 2122 (2001).

${ }^{24}$ A. O’Riordan, R. Van Deun, E. Mairiaux, S. Moynihan, P. Fias, P. Nockemann, K. Binnemans, and G. Redmond, Thin Solid Films, 516, 5098 (2008).

${ }^{25}$ A. O’Riordan, E. O’Connor, S. Moynihan, P. Nockemann, P. Fias, R. Van Deun, D. Cupertino, P. Mackie, and G. Redmond, Thin Solid Films, 497, 299 (2006). 
${ }^{26}$ Y. Kawamura, Y. Wada, Y. Hasegawa, Mi. Iwamuro, T. Kitamura, and S. Yanagida, Appl. Phys., Lett., 74, 3245 (1999).

${ }^{27}$ O.M. Kheris, R, J. Curry, M. Somerton, and W. P. Gillin, J. Appl. Phys. 88, 777 (2000).

${ }^{28}$ Z.-Q. Chen, F. Ding, Z.-Q. Bian, and C.-H. Huang, Org. Electron. 11, 369 (2010).

${ }^{29}$ R. Nagata, H. Nakanotani, and C. Adachi, Adv. Mater. 29, 1604265 (2016).

${ }^{30}$ A. Graf, C. Murawski, Y. Zakharko, J. Zaumseil, and M. C. Gather, Adv. Mater. 30, 1706711 (2018).

${ }^{31}$ H. Xu, Q. Sun, Z. An, Y. Wei, and X. Liu, Coord. Chem. Rev. 293-294, 228 (2015).

${ }^{32}$ C. Adachi, M. A. Baldo, S. R. Forrest, J. Appl. Phys. 87, 8049 (2000).

${ }^{33}$ T. W. Canzler, J. Kido, Org. Electron. 7, 29 (2006).

${ }^{34}$ S. Zhang, G. A. Turnbull, I. D. W. Samuel, Org. Electron. 13, 3091 (2012).

${ }^{35}$ J. Mezyk, D. Di Nuzzo, A. Mech, R. Tubino, and F. Meinardi, J. Chem. Phys. 132, 024504 (2010).

${ }^{36}$ M. A. Baldo, C. Adachi, and S. R. Forrest, Phys. Rev. B 62, 10967 (2000).

${ }^{37}$ K. Hayashi, H. Nakanotani, M. Inoue, K. Yoshida, O. Mikhnenko, T.-Q. Nguyen, and C. Adachi, Appl. Phys. Lett. 106, 093301 (2015).

${ }^{38}$ S. Reineke, G. Schwartz, K. Walzer, and K. Leo, Appl. Phys. Lett. 91, 123508 (2007).

${ }^{39}$ G. He, M. Pfeiffer, K. Leo, M. Hofmann, J. Birnstock, R. Pudzich, and J. Salbeck, Appl. Phys. Lett. 85, 3911 (2004).

${ }^{40}$ S. Ying, D. Yang, X. Qiao, Y. Dai, Q. Sun, J. Chen, T. Ahamad, S. M. Alshehri, and D. Ma, J. Mater. Chem. C 6, 10793 (2018).

${ }^{41}$ B. Zhao, H. Zhang, Y. Miao, Z. Wang, L. Gao, H. Wang, Y. Hao, B. Xuab, and W. Li, J. Mater. Chem. C 5, 12182 (2017).

${ }^{42}$ Y. Yin, X. Piao, Y. Li, Y. Wang, J. Liu, K. Xu, and W. Xiea, Appl. Phys. Lett. 101, 063306 (2012).

${ }^{43}$ J. Lee, J.-I. Lee, J. Y. Lee, and H. Y. Chu, Appl. Phys. Lett. 94, 193305 (2009).

${ }^{44}$ Q. Xin, W. L. Li, G. B. Che, W. M. Su, X. Y. Sun, B. Chu, and B. Li, Appl. Phys. Lett. 89, 223524 (2006). 


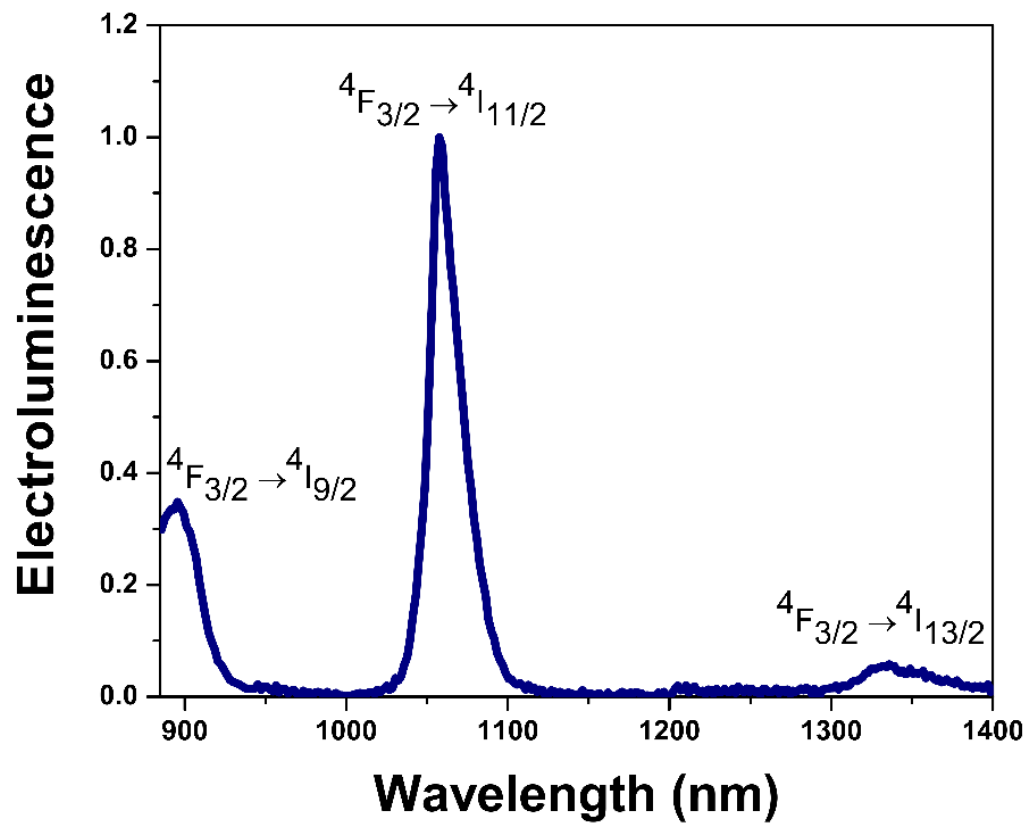

FIG. 1. Normalized NIR electroluminescence spectrum of the device recorded at $15 \mathrm{~V}$.

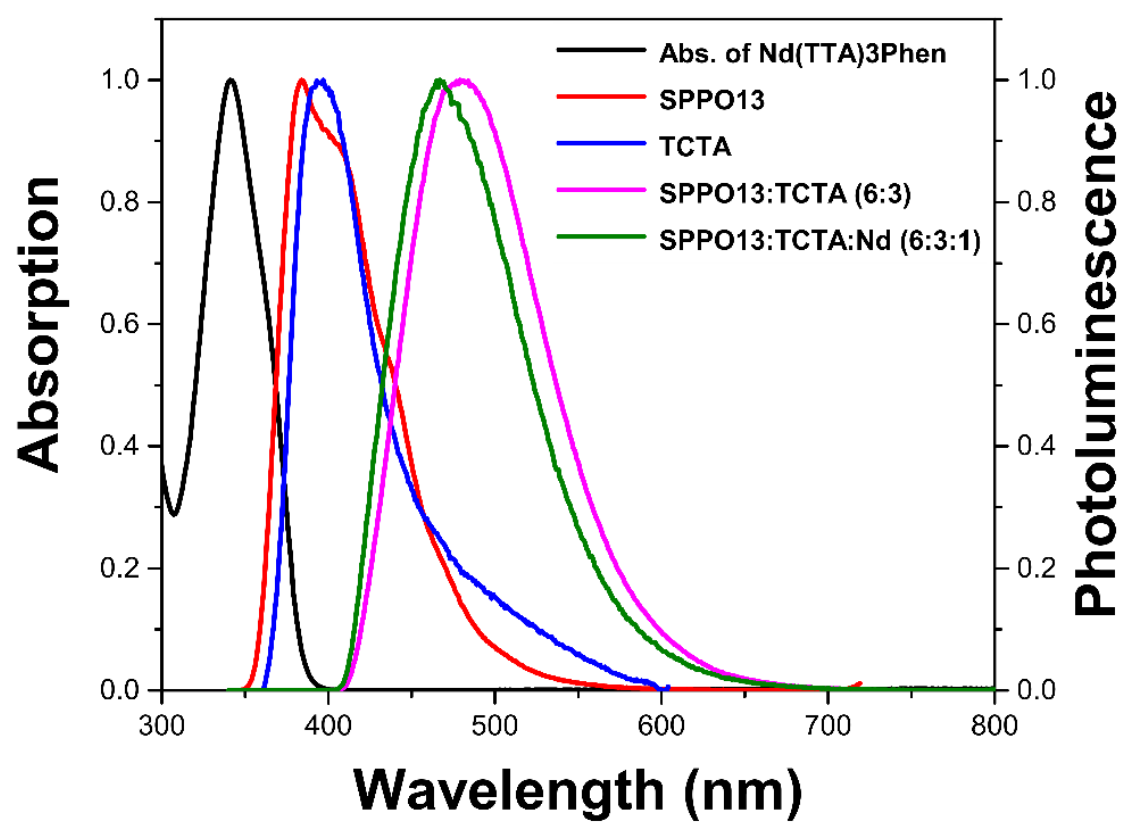

FIG. 2. Normalized absorption spectrum of $\mathrm{Nd}(\mathrm{TTA})_{3} \mathrm{Phen}$ recorded from dichloromethane solution, and steady-state photoluminescence spectra of SPPO13, TCTA, SPPO13:TCTA (6:3 weight ratio), and SPPO13:TCTA:Nd(TTA) 3 Phen (6:3:1 weight ratio) recorded from spin-coated films on quartz substrates. 


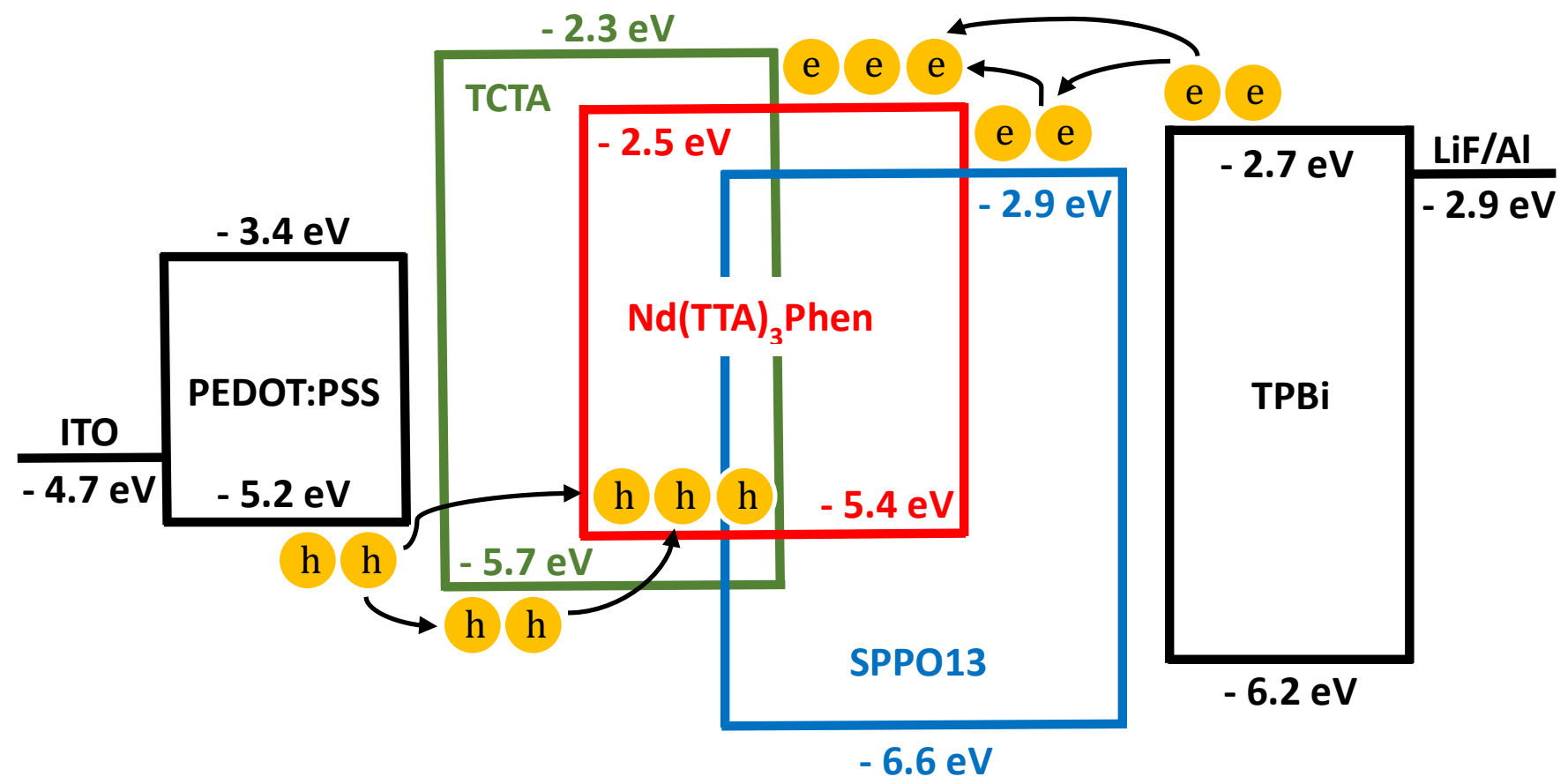

FIG. 3. Energy level alignment of the materials in the device structure. Charge transport pathways and the excitation of $\mathrm{Nd}(\mathrm{TTA})_{3}$ Phen through the ETL and HTL and also direct charge trapping are shown. 

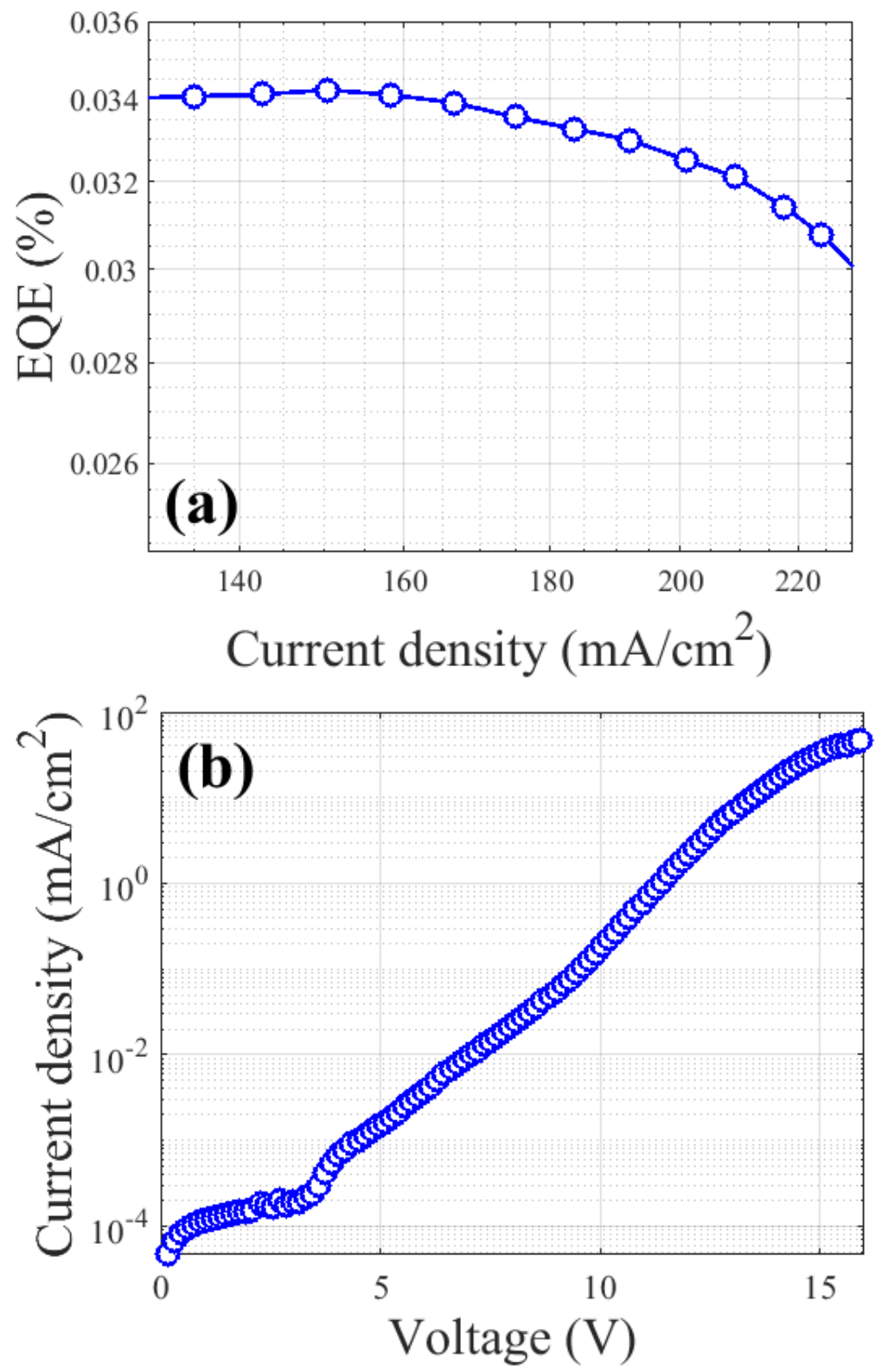

FIG. 4. (a) EQE vs. current density, and (b) current density vs. voltage plots for the device. 